\title{
How Often Must We Apply Syndrome Measurements?
}

\author{
Y. S. Weinstein \\ Quantum Information Science Group, MITRE, 200 Forrestal Rd., Princeton, NJ 08540
}

\begin{abstract}
Quantum error correction requires encoding quantum information into a quantum error correction code and measuring error syndromes to detect and identify possible errors. Quantum fault tolerance typically assumes that syndrome measurements are applied after every logical gate at great expense both in time and number of qubits. Here we demonstrate that not only is this not necessary, but that we may achieve greater accuracy when applying syndrome measurements less often. Our simulations are performed within the [[7,1,3]] quantum error correction code but may be applicable to a broad range of codes.
\end{abstract}

Keywords: syndrome measurements, quantum error correction, quantum fault tolerance

\section{INTRODUCTION}

Quantum error correction (QEC) ${ }^{1-3}$ is an essential component of hoped for realistic quantum computers. QEC consists of three basic parts: encoding quantum information into a QEC code, measuring error syndromes to detect and identify the error that occurred and, if an error did occur, applying an appropriate recovery operation. Syndrome measurements (SM) are applied often at great expense in terms of implementation time and number of qubits. In fact, standard approaches to quantum fault tolerance (QFT), the computational framework that will allow for successful quantum computation despite a finite probability of error in basic computational gates, ${ }^{4-7}$ assumes SM are applied after every operation. Here, we demonstrate that applying SM after every operation is not necessary and in fact should not be done. Applying SM less often can provide immense savings in time and qubits and will generally output a more accurate final state. We support our assertion via numerical simulations of multiple single-logical-qubit operations on a qubit of quantum information encoded in the $[[7,1,3]]$ QEC code. ${ }^{8}$

In order to keep quantum information stored in a QEC code protected from error we must implement logical gates within the encoding. This greatly restricts the possible gates that can be performed. Nevertheless, universal quantum computation can be performed within many QEC codes if we utilize the gate set Clifford gates plus the $T$-gate, a singlequbit $\pi / 4$ phase rotation. For Calderbank-Shor-Steane (CSS) codes such as the [[7,1,3]] code, the 'logical' Clifford gates can be implemented bit-wise, but 'logical' $T$-gates requires interaction between the data qubits and a specified ancilla state.

To implement an arbitrary operation we must determine the proper combination of Clifford and $T$-gates. The question of performing a single-qubit rotation (within prescribed accuracy) was originally examined in ${ }^{9,10}$ and is now an area of intense investigation. ${ }^{11-17}$ The primary goal of these explorations has been to design circuits within $\epsilon$ of a desired (arbitrary) rotation while limiting the utilization of resource-heavy $T$-gates. As an example, $R_{Z}(.1)$ can be implemented with accuracy better than $10^{-5}$ using $78^{16}$ or $56^{17} T$-gates, interspersed by at least as many single-qubit Clifford gates. QFT would suggest that SM be applied after each of the more than 100 gates needed at a cost of thousands of additional qubits and hundreds of time steps.

The tenets of QFT guarantee successful quantum computation, but at the cost of a great number of qubits and gates. Recent work has explored the consequences of relaxing some of these strict rules while still achieving high fidelity gates. ${ }^{18-20}$ A few studies have specifically addressed the question of performing SM less often than after every gate. ${ }^{21-25}$ They demonstrate that applying SM less often will consume less resources, while still enabling successful quantum computation and, in general, achieving higher fidelity.

The following argument demonstrates that there is no fundamental need to apply SM after every logical gate. Let us use as our example the $[[7,1,3]]$ QEC code. Measuring the syndromes of this code will allow us to correctly identify and

Further author information: (Send correspondence to Y. S. Weinstein)

Y. S. Weinstein: E-mail: weinstein@mitre.org 
correct an error on a single physical qubit. Were errors to have occurred on two (physical) qubits the SM will (generally) misidentify the error. For this example, we begin with a single qubit state perfectly encoded in the $[[7,1,3]]$ QEC code. We then apply Clifford gates that are imperfectly implemented: causing an error on each qubit with probability $p \ll 1$. After one Clifford gate the probability that an error occurred on one qubit is then $7 p-\mathcal{O}\left(p^{2}\right)$. The probability that an error occurred on two qubits is $21 p^{2}-\mathcal{O}\left(p^{3}\right)$. Given that $p$ is small we can reasonably assume that, at most, only one qubit will have an error. That error can be identified by SM and corrected by an appropriate recovery operation. If we were now to implement a second Clifford gate, without performing SM in between, the probability that an error occurred to one qubit increases to $14 p-\mathcal{O}\left(p^{2}\right)$ and the probability that errors occurred to two qubits increases to $84 p^{2}-\mathcal{O}\left(p^{3}\right)$. Again, the probability of errors on two qubits is second order in $p$ and if we were to now apply SM and a recovery operation we will almost certainly correct the state of the system. After $n$ gates without SM the probability of an error on one qubit is $7 n-\mathcal{O}\left(p^{2}\right)$ and on two qubits $21\left(n+2\left(\begin{array}{l}n \\ 2\end{array}\right)\right) p^{2}$. Yet again, the probability that two errors occur remains of order $p^{2}$ and SM will correct the single-qubit errors. Given that QEC will correct an error of one qubit and the probability of errors on two qubits is small clearly QEC is not needed until the end of the gate sequence. This is because at no point will the probability of errors on two or more qubits be of order $p$ (a similar argument can be made when including a two-qubit Clifford gate such as a controlled-NOT gate, this will be explored elsewhere). $T$-gates are not implemented bit-wise. Nevertheless, as we demonstrate below, the rules of QFT ensure that the probability of an error occurring on two qubits is of order $p^{2}$. Thus, even if our gate sequence includes $T$-gates, assuming the error probability is small enough, there is no reason to apply SM until the end of the entire sequence.

So why is it assumed that SM are applied often? The reason is that our error probability is not that low. If we do not apply SM often enough the probability that errors will occur on two qubits will become too high. This will either cause the SM at the end of the gate sequence to be too high, or will cause strong degredation of fidelity even if the SM may be successful. Of course, if SM were implemented perfectly and we had infinite resources, we should apply SM as often as possible. Given that this is unrealistic we are left to ask, how often should SM be applied? Constant application of SM will be costly in time and qubits and, because they are imperfect, may introduce additional errors. Not applying SM often enough may allow error probabilities to grow too quickly.

Our explorations will be conducted via simulations of logical single-qubit gates performed on quantum information encoded in the [[7,1,3]] QEC code in a nonequiprobable Pauli operator error environment ${ }^{26}$ with non-correlated errors. In this error model different Pauli errors occur with different arbitrary probabilities. Each individual physical qubit that takes part in a gate, measurement, or initialization undergoes a $\sigma_{x}^{j}$ error with probability $p_{x}, \sigma_{y}^{j}$ error with probability $p_{y}$, and $\sigma_{z}^{j}$ error with probability $p_{z}$, where $\sigma_{i}^{j}, i=x, y, z$ are the Pauli spin operators on qubit $j$. Qubits not taking part in a gate operation, initialization, or measurement are assumed to be perfectly stored.

In our simulations we begin with an arbitrary single-qubit state, $|\psi\rangle=\cos \alpha|0\rangle+e^{i \beta} \sin \alpha|1\rangle$, perfectly encoded into the [[7,1,3]] error correction code. We then apply of sequence of gates. The sequence is made up of composite gates $A=H P T$ and $B=H T$, where $H$ is the Hadamard gate and $P$ is a phase gate. These composite gates are form the basic building blocks for longer gate sequences capable of implementing arbitrary single qubit gates. The exact simulations of each gates is as follows. Implementing a Clifford gate, $C$ on the $[[7,1,3]]$ QEC code requires implementing $C^{\dagger}$ on each of the 7 qubits. The first step in performing the logical $T$-gate is the construction of the ancilla state $|\Theta\rangle=\frac{1}{\sqrt{2}}\left(\left|0_{L}\right\rangle+e^{i \frac{\pi}{4}}\left|1_{L}\right\rangle\right)$, where $\left|0_{L}\right\rangle$ and $\left|1_{L}\right\rangle$ are the logical basis states on the [[7,1,3]] QEC code. Bit-wise CNOT gates are then applied between the state $|\Theta\rangle$ and the encoded state with the $|\Theta\rangle$ state qubits as control. Measurement of zero on the encoded state projects onto the qubits that had made up the $|\Theta\rangle$ state the encoded state with the application of a $T$-gate.

To ensure fault tolerance in the construction of $|\Theta\rangle$ requires the following steps: (1) A logical zero state is encoded by applying error correction to 7 qubits all initially in the state $|0\rangle{ }^{4}$ We use Shor state ancilla for syndrome measurements. ${ }^{5}$ (2) A seven qubit Shor state ${ }^{5}$ in constructed and proper verifications are applied. (3) Seven controlled-ZPX gates:

$$
C(Z P X)=\left(\begin{array}{cccc}
1 & 0 & 0 & 0 \\
0 & 1 & 0 & 0 \\
0 & 0 & 0 & e^{i \frac{\pi}{4}} \\
0 & 0 & e^{-i \frac{\pi}{4}} & 0
\end{array}\right),
$$

are applied each between a qubit of the Shor state and a qubit of the logical zero state with the Shor state qubits as control. (4) Measurement of the Shor state (with even parity outcome) completes the projection and the construction of the logical state $|\Theta\rangle$. Circuits for these steps are shown in Figs. 1 and 2. Our simulations follow the method described in. ${ }^{20}$ 


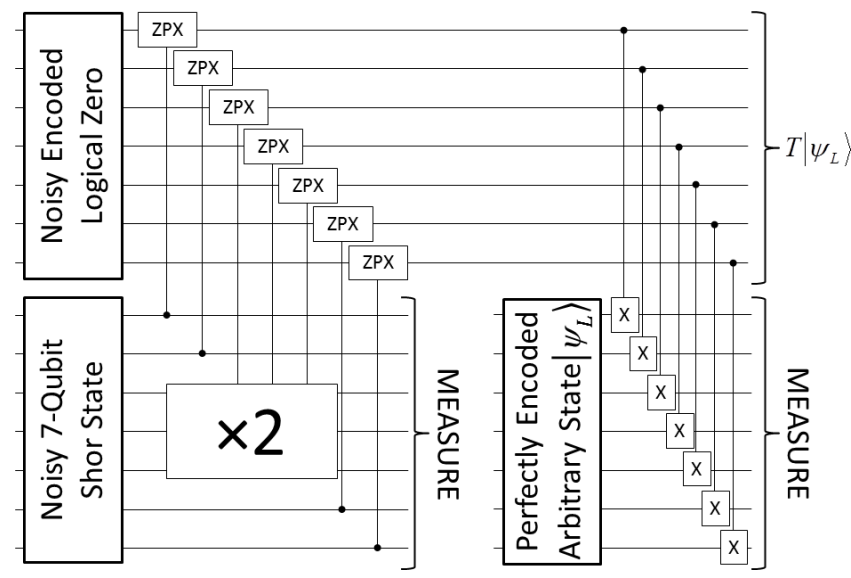

Figure 1. Implementation of [[7,1,3]] QEC code $T$-gate.

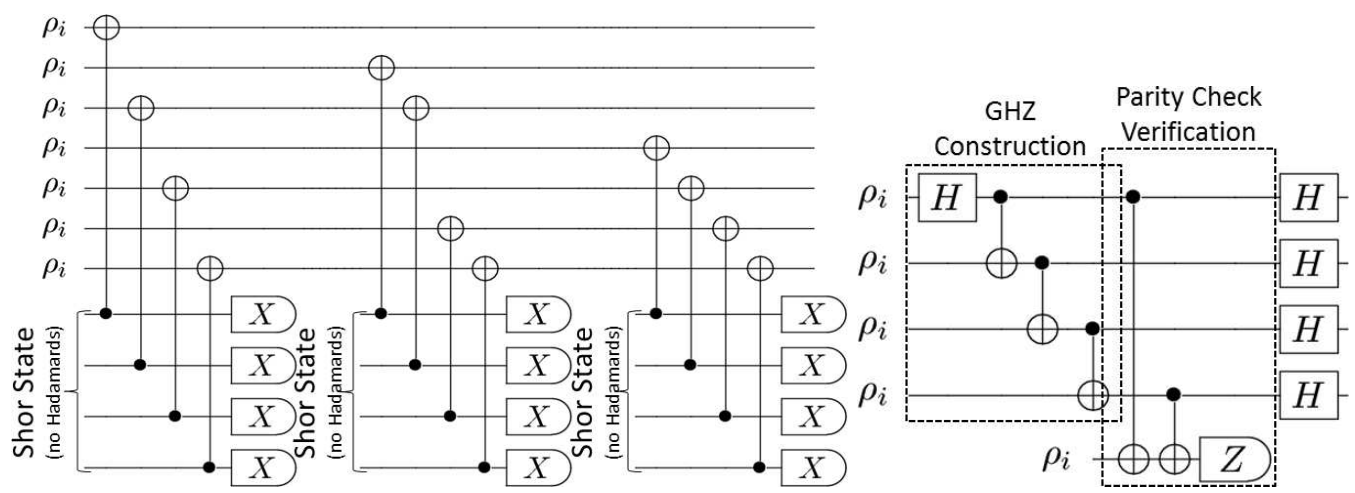

Figure 2. Left: Circuit for phase syndrome measurement on the [[7,1,3]] QEC code. Right: Four qubit Shor state construction with one verification.

SM are performed via the Shor state ancilla. Shor states appropriate for the [[7,1,3]] QEC code are four qubit GHZ states with a Hadamard gate appended for each qubit. The construction of the necessary Shor states is now described and, in our simulations, is done in the non-equiprobable error environment. We start with four qubits assumed to be noisily initialized in the state $\left|\psi_{i}\right\rangle=\frac{1}{\sqrt{2}}(|0\rangle+|1\rangle)|000\rangle$. The construction requires three CNOT gates and a verification step to test the parity of the qubits and ensure no error has taken place. The construction is shown in Fig. 2.

As shown in Fig. 2 bit-flip and phase-flip SM each require three measurements to properly locate and identify potential errors. In order to adhere to the rules of QFT, these six SM must be repeated until the same SM is read out twice to ensure that no errors happened during the SM themselves. In our simulations we have assumed that the same SM is read out the first two times. In addition, we assume that the SM indicates no error has occurred. Were the SM to indicate error, a (noisy) recovery operation would have to be applied with some cost to the accuracy of the implementation.

To determine how often SM should be applied during a gate sequence we simulate the implementation of 20 randomly chosen composite gates (comprising 50 total gates):

$$
U=A B B B A A A A B B A B A B A B B B A A,
$$

with each gate $H, P$, and $T$ and all associated ancilla construction, implemented in the non-equiprobable error environment. We then formulate 7 SM application schemes: applying QEC after every gate, $(q=50$, where $q$ is the number of QEC applications), after every composite gate $A$ and $B(q=20)$, after every two composite gates $(q=10)$, after every 5 composite gates $(q=4)$, after each half of the sequence $U(q=2)$, after the entire sequence $(q=1)$, and not at all $(q=0)$. Every physical gate of the QEC implementation, including Shor state construction, ${ }^{27}$ is also done in the nonequiprobable error environment. Each scheme is simulated for error environments of different values of $p_{x}, p_{y}$ and $p_{z}$. Our initial state is the basis state $|0\rangle$. Other initial states and gate sequences were explored and give similar results. 
Table 1. Second line: infidelity of final state after 50 noisy gates with noisy SM applied after each as a function of depolarization strength $p=p_{x}=p_{y}=p_{z}$. Lower lines: fractional increase or decrease of infidelity for different SM application schemes compared to SM after every gate.

\begin{tabular}{|c|c|c|c|c|}
\hline $\mathrm{q}$ & $p=10^{-9}$ & $p=10^{-7}$ & $p=10^{-5}$ & $p=10^{-3}$ \\
\hline \hline 50 & $4.50 \times 10^{-8}$ & $4.50 \times 10^{-6}$ & $4.54 \times 10^{-4}$ & $8.27 \times 10^{-2}$ \\
\hline \hline 20 & $1.46 \times 10^{-7}$ & $7.52 \times 10^{-7}$ & $7.55 \times 10^{-5}$ & $7.85 \times 10^{-3}$ \\
\hline 10 & $1.62 \times 10^{-7}$ & $2.73 \times 10^{-7}$ & $2.80 \times 10^{-5}$ & $4.97 \times 10^{-3}$ \\
\hline 4 & $2.34 \times 10^{-7}$ & $-1.97 \times 10^{-7}$ & $-1.89 \times 10^{-5}$ & $1.52 \times 10^{-3}$ \\
\hline 2 & $2.20 \times 10^{-7}$ & $-2.73 \times 10^{-7}$ & $-2.65 \times 10^{-5}$ & $9.88 \times 10^{-4}$ \\
\hline 1 & $2.96 \times 10^{-7}$ & $-3.41 \times 10^{-7}$ & $-3.32 \times 10^{-5}$ & $5.12 \times 10^{-4}$ \\
\hline 0 & -1.02 & -1.02 & -1.01 & -.544 \\
\hline
\end{tabular}

Each of the SM application schemes will be compared for cost and accuracy. The cost is straightforward. Each SM application comes at a cost of 12 Shor states totalling at least 60 qubits (4 per state and one for verification) and at least 12 time steps. We leading to a final state, $\rho_{f}$, of the 7 qubits. We determine the accuracy of the simulated implementations by comparing the final seven-qubit state, $\rho_{f}$, with the final state after perfectly applied gates, $\rho_{i}$ via the state fidelity $F\left(\rho_{i}, \rho_{f}\right)=\operatorname{Tr}\left[\rho_{i} \rho_{f}\right]$. In addition we will find it useful to utilize the infidelity $I\left(\rho_{i}, \rho_{f}\right)=1-F\left(\rho_{i}, \rho_{f}\right)$. We note that this fidelity measure gives very similar results to the single logical qubit fidelity because the errors affect the entire state space more or less uniformly.

In this paper we look concentrate on a depolarizing environment $p=p_{x}=p_{y}=p_{z}$. Other environments are explored elsewhere. ${ }^{24,25}$ Results of our simulations are shown in the Table. The second line of Table reports the infidelity of the output state when QEC is applied after every gate, $I_{50}$. The lower lines show the fractional change, $D$, of the infidelity when using other QEC application schemes:

$$
D\left(I_{50}, I_{q}\right)=\frac{I_{50}-I_{q}}{I_{50}}
$$

for $q=20,10,4,2,1,0$. A positive fractional change means a higher fidelity when using less QEC. Negative fractional change means the fidelity is higher when applying QEC after every gate. We note, however, that even if applying QEC after every gate gives the highest fidelity, this does not mean it is the optimal choice of QEC application scheme. If the fractional change, $D\left(I_{50}, I_{1}\right)$ is small one may achieve an almost optimal fidelity while saving a factor of up to 50 in time and number of qubits, perhaps a worthwhile tradeoff.

From the Table we see that the optimum accuracy is achieved by applying SM after every composite gate $(q=20)$. This also provides a $60 \%$ savings in time and number of qubits when compared to applying SM after every gate. The case of $q=10$ also provides better accuracy than applying SM after every gate. This demonstrates that applying (noisy) SM more often does not translate to higher fidelity. For $q<10$ we find that the fidelity is lower than when applying SM after every gate. Nevertheless, the reduction in accuracy by a few parts in $10^{6}$ may be worthwhile given that it comes with a savings in resources of up to $98 \%$.

We now look at the infidelities themselves as a function of error probabilites and number of gates. We concentrate on the case when QEC is applied after every gate and note that deviations from this for the other QEC schemes are extremely small. In a depolarizing environment the infidelity increases by an order of magnitude for every order of magnitude increase in error probability up to $p=10^{-4}$, after which the increase is slightly faster.

In a depolarizing environment when QEC is applied after every gate, the infidelity increases linearly as a function of gate number as shown in Fig. 3. When QEC is not applied after every gate, the infidelity is lowest after a $T$ gate and increases after application of an $H$ or $P$ gate. This behavior will be explored elsewhere. When QEC is applied the infidelity is lowered practically to that of the scheme when QEC is applied after every gate. The linear fit for infidelity for an error probability of $p=10^{-3}$ is given by $8.1 \times 10^{-4} t+.045$ where $t$ is the gate number. For $p=10^{-5}, 10^{-7}$, and $10^{-9}$, the fits are given by $8.3 \times 10^{-8} t+4.5 \times 10^{-4}, 8.3 \times 10^{-12} t+4.5 \times 10^{-5}$, and $8.3 \times 10^{-16} t+4.5 \times 10^{-7}$. To achieve better insight, we define a critical fidelity, $F_{c}$. Output states with lower fidelity will be considered unacceptable. Based on the fits we can determine how many gates can be performed while the output state fidelity remains below $F_{c}$. Assuming $F_{c}=.9$ we find that for error probability $p=10^{-3} 69$ gates can be performed. For $p=10^{-5}, 10^{-7}$, and $10^{-9}$, the number of 


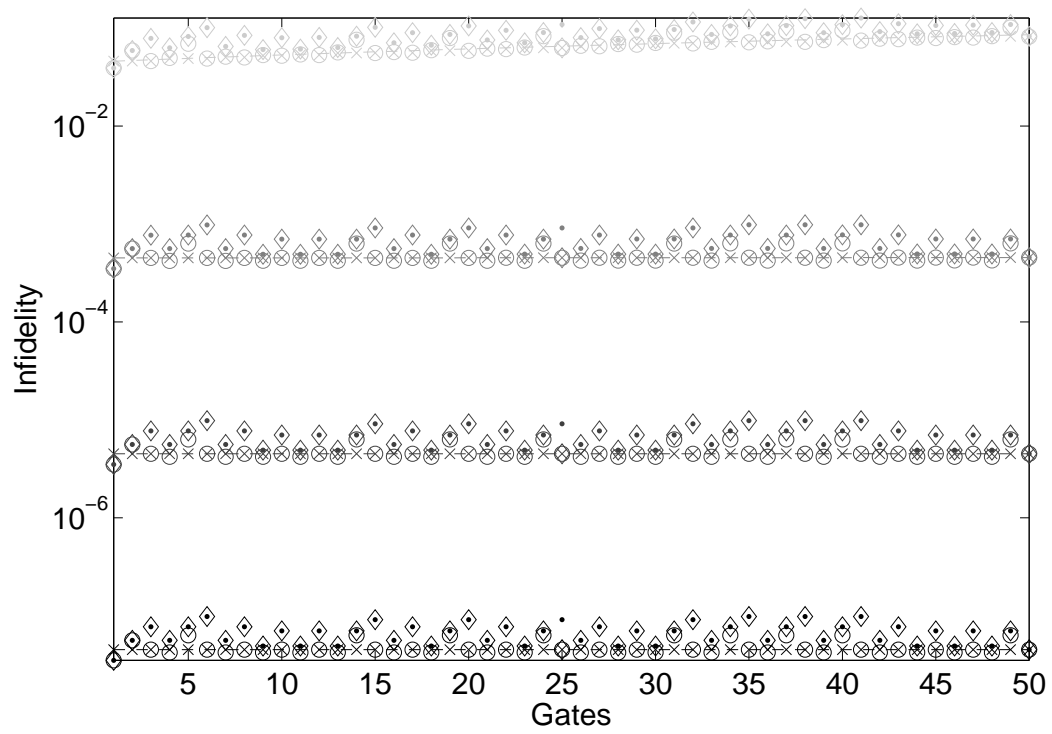

Figure 3. Fidelity as a function of gates in the depolarizing environment for $p=10^{-3}, 10^{-4}, 10^{-5}, 10^{-6}$ (top to bottom) and for QEC implementation methods $q=1(\cdot), q=2(\diamond), q=20(\circ), q=50(\times)$. The dashed line is a least squares linear fit to fidelity when $q=50$.

gates is $1.1 \times 10^{7}, 1.1 \times 10^{1} 1$, and $2.5 \times 10^{1} 5$. In other words, for every order of magnitude decrease in error probability, we find about a two order magnitude increase in the number of gates that can be performed for a given $F_{c}$ (similar trends occur for other values of $F_{c}$ ).

In conclusion, our study casts doubt on the assumption that one should apply SM after every logical gate. Our simulations demonstrate that applying Shor state SM on information encoded in the [[7,1,3]] QEC code after every logical gate will generally not maximize the output state fidelity. In a depolarizing environment it is best to apply QEC after every composite $A$ and $B$ gate. In addition, the difference in fidelity between the scheme where QEC is applied after every gate and a scheme where QEC is applied only once after 50 gates is extremely minimal. It may be far more practical to apply SM less often and gain a 50-fold savings in time and number of qubits.

Our analysis points to additional trends that are important for aspects of quantum fault tolerance. First, we have determined the change in fidelity for the depolarizing environment as a function of error probability. Importantly we have calculated the number of gates that can be applied in a depolarizing environment before reaching a given critical fidelity and shown how that number changes with error strength. Finally, we have seen how the fidelity changes with time depending on which gates and when SM are applied. While these simulations were done with a specific choice of QEC code and SM the results promise to be important for explorations of other QEC choices. Further work will explore other QEC codes and strategies with the goal of tailoring an optimal error correction and SM approach to a given error environment.

\section{Acknowledgment}

I would like to acknowledge support from the MITRE Innovation Program.

\section{REFERENCES}

[1] M Nielsen and I. Chuang, Quantum Information and Computation (Cambridge University Press, Cambridge, 2000). [2] P.W. Shor, Phys. Rev. A 52, R2493 (1995).

[3] A.R. Calderbank and P.W. Shor, Phys. Rev. A 54, 1098 (1996); A.M. Steane, Phys. Rev. Lett. 77, 793 (1996).

[4] J. Preskill, Proc. Roy. Soc. Lond. A 454, 385 (1998). 
[5] P.W. Shor, Proceedings of the the 35th Annual Symposium on Fundamentals of Computer Science,(IEEE Press, Los Alamitos, CA, 1996).

[6] D. Gottesman, Phys. Rev. A 57, 127 (1998).

[7] P. Aleferis, D. Gottesman, and J. Preskill, Quant. Inf. Comput. 6, 97 (2006).

[8] A. Steane, Proc. Roy. Soc. Lond. A 452, 2551 (1996).

[9] A.Y. Kitaev, Russ. Math. Surv. 52, 1191 (1997); A.Y. Kitaev, A.H. Shen, and M.N. Vyalyi, Classical and Quantum Computation Graduate Studies in Mathematics 47, American Mathematical Society 2002.

[10] C.M. Dawson and M.A. Nielsen, Quant. Inf. Comp. 6, 81 (2006).

[11] A. Bocharov and K.M. Svore, Phys. Rev. Lett. 109, 190501 (2012).

[12] V. Kliuchnikov, D. Maslov, and M. Mosca, Quant. Inf. Comp. 13, 607 (2013).

[13] T.T. Pham, R. Van Meter, and C. Horsman, Phys. Rev. A 87, 052332 (2013).

[14] G. Duclos-Ciani and K.M. Svore, arXiv:1210.1980.

[15] V. Kliuchnikov, D. Maslov, and M. Mosca, Phys. Rev. Lett. 110, 190502 (2013).

[16] P. Selinger, arXiv:1212.6253.

[17] V. Kliuchnikov, D. Maslov, and M. Mosca, arXiv:1212.6964.

[18] Y.S. Weinstein, Phys. Rev. A 84, 012323 (2011).

[19] S.D. Buchbinder, C.L. Huang, and Y.S. Weinstein, Quant. Inf. Proc. 12, 699 (2013).

[20] Y.S. Weinstein, Phys. Rev. A 87, 032320 (2013).

[21] M.G. Whitney, N. Isailovic, Y. Patel, and J. Kubiatowicz, Proceedings of the 36th Annual International Symposium on Computer Architecture, (ACM, New York, 2009).

[22] A.A. Nada, B. Fortescue, and M. Byrd, arXiv:1303.4026.

[23] Y. Tomita, et. al, arXiv:1305.0349.

[24] Y.S. Weinstein, Phys. Rev. A 88, 012325 (2013).

[25] Y.S. Weinstein, Phys. Rev. A 89, 020301(R) (2014).

[26] V. Aggarwal, A.R. Calderbank, G. Gilbert, Y.S. Weinstein, Quant. Inf. Proc. 9, 541 (2010).

[27] Y.S. Weinstein and S.D. Buchbinder, Phys. Rev. A 86, 052336 (2012). 